\title{
烯丙型醇与取代肼的对映专一性烯丙型烷基化反应
}

\author{
徐靖坤谷永红* 田仕凯*
}

(中国科学技术大学化学系 合肥 230026)

\begin{abstract}
摘要 发展了首例烯丙型醇与取代肼的对映专一性烯丙型烷基化反应. 在钯/外消旋 2,2'-双 (二苯膦基)-1,1'-联䒺 (BINAP)/硼酸的催化下，一系列高度对映富集的烯丙型醇与取代肼于室温进行烯丙型烷基化反应，具有高度的区域选 择性, 立体构型的保留率大于 $95 \%$, 适于合成高对映纯度的烯丙型肼. 另外, 该反应的唯一副产物是水, 对环境友好.

关键词 醇; 肼; 烯丙型烷基化; 钯; 对映专一性
\end{abstract}

\section{Enantiospecific Allylic Alkylation of Substituted Hydrazines with Allylic Alcohols}

\author{
$\mathrm{Xu}$, Jing-Kun $\quad \mathrm{Gu}$, Yonghong* Tian, Shi-Kai* \\ (Department of Chemistry, University of Science and Technology of China, Hefei 230026)
}

\begin{abstract}
Unprecedented enantiospecific allylic alkylation of substituted hydrazines with allylic alcohols has been developed. A range of substituted hydrazines underwent palladium/racemic 2,2'-bis(diphenylphosphino)-1,1'-binaphthyl (BINAP)/boric acid-catalyzed allylic alkylation with highly enantioenriched allylic alcohols at room temperature and the reaction proceeded in a highly regioselective fashion with greater than $95 \%$ retention of configuration to afford allylic hydrazines with high enantiopurity. Moreover, only water was generated as an environmentally benign byproduct.
\end{abstract}

Keywords alcohols; hydrazines; allylic alkylation; palladium; enantiospecificity

Tsuji-Trost 反应，即钯催化的烯丙型烷基化反应， 是构建碳一碳键和碳一杂原子键的重要方法 ${ }^{[1]}$, 常以醋酸 烯丙酯、碳酸烯丙酯、磷酸烯丙酯、烯丙卤等作为烯丙 型亲电试剂, 而这些试剂通常由相应的烯丙型醇衍生化 而得, 因此直接以烯丙型醇为烯丙型亲电试剂的相应反 应越来越受到人们的重视 ${ }^{[2]}$. 在 Tsuji-Trost 反应中直接 利用烯丙型醇不仅可以缩短对同一目标产物的合成路 线, 而且被取代的羟基的质量仅为 $17 \mathrm{Da}$, 远低于常用 烯丙型亲电试剂中离去基团(乙酰氧基、烷氧酰氧基、 卤素等)的质量, 因而可以大幅度地提高反应的原子经 济性. 虽然如此, 但羟基的离去能力很差, 对官能团的 容忍范围较小, 因而在 Tsuji-Trost 反应中烯丙型醇的直 接利用具有很大的挑战性.

对映富集的烯丙型亲电试剂在 Tsuji-Trost 反应中生 成的手性 $\pi$-烯丙基钯中间体很容易发生消旋化, 因而难
以将底物的对映纯度完全传递给产物 ${ }^{[1]}$. 尽管如此, 对 映富集的二级烯丙型醇在适当的反应条件下可以与一 些碳 ${ }^{[3]} 、$ 氮 ${ }^{[4]}$ 、氧 ${ }^{[5]}$ 、硫 ${ }^{[6]}$ 亲核试剂进行对映专一性烯丙 型烷基化反应. 由于对映富集的二级烯丙型醇容易通过 动力学拆分、不对称催化羰基加成等多种方法来制备 ${ }^{[7]}$, 因此这样的手性转移反应具有很高的合成应用价值.

在 2013 年, Mäeorg 等报道了一级烯丙型醇与单、 双取代肼在双(二亚苄基丙酮)钯 $\left[\mathrm{Pd}(\mathrm{dba})_{2}\right] / 1,1^{\prime}$-双 (二苯 膦基)二茂铁( $\mathrm{dppf}) / \mathrm{P}(\mathrm{OPh})_{3}$ 催化下的烯丙型烷基化反应, 但没有涉及到二级烯丙型醇的反应以及手性烯丙型肼 的构建 (Scheme 1) ${ }^{[8]}$. 手性肼不仅在药物中有重要应用 价值 ${ }^{[9]}$, 还被广泛用于杂环合成、偶联反应以及不对称 合成反应 ${ }^{[10]}$, 特别是手性烯丙型肼可以进行一些有趣 的重排反应 ${ }^{[11]}$. 然而, 制备手性烯丙型肼的方法非常有 限. 虽然不对称催化的 Tsuji-Trost 反应已被用于手性烯

\footnotetext{
*E-mail: ygu01@ustc.edu.cn, tiansk@ustc.edu.cn

Received December 29, 2014; revised January 17, 2015; published online January 20, 2015.
}

Project supported by the National Natural Science Foundation of China (Nos. 21472178 and 21232007), the National Key Basic Research Program of China (No. 2014CB931800), and the Natural Science Foundation of Anhui Province of China (No. 1408085MB24).

国家自然科学基金(Nos. 21472178 和 21232007)、国家重点基础研究发展计划(No. 2014CB931800)和安徽省自然科学基金(No. 1408085MB24)资助项 目. 


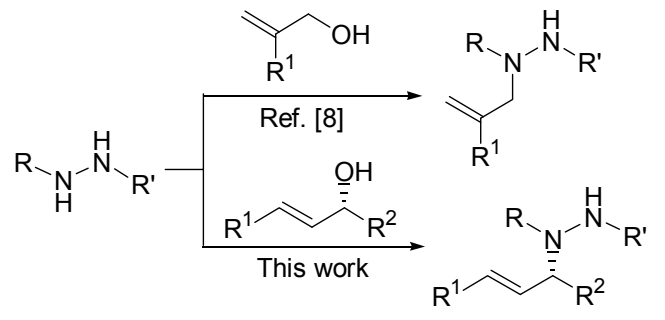

图式 1 烯丙型醇与取代肼的烯丙型烷基化反应

Scheme 1 Allylic alkylation of substituted hydrazines with allylic alcohols

丙型肼的合成，但仅限于 $\alpha$-取代基与 $\gamma$-取代基相同的烯 丙型底物 ${ }^{[12]}$. 我们在探索原子经济性取代反应的过程 中 $^{[13]}$, 发展了一级烯丙型胺与取代肼的对映专一性烯 丙型烷基化反应，可以合成手性非对称烯丙型肼(其 $\alpha$ 取代基不同于 $\gamma$-取代基 $)^{[14]}$. 此外, 偶氮二羧酸酯的不对 称催化加成反应也可以合成一些特定类型的手性烯丙 型胅 ${ }^{[15]}$. 为了拓展手性烯丙型肼的合成方法, 我们基于 Mäeorg 等的报道 ${ }^{[8]}$, 结合对原子经济性取代反应 ${ }^{[13]}$ 以 及单取代肼 ${ }^{[16]}$ 的研究兴趣, 发展了 $[\mathrm{Pd}(\mathrm{allyl}) \mathrm{Cl}]_{2} /$ 外消旋 $2,2^{\prime}$-双 (二苯膦基)-1,1'-联菜(BINAP)/嗍酸催化的二级烯 丙型醇与取代肼的对映专一性烯丙型烷基化反应, 合成 一系列高对映纯度的烯丙型肼(Scheme 1). 下面报道我 们的研究结果.

\section{1 结果与讨论}

我们首先将 Mäeorg 等发展的反应条件 $\left[\mathrm{Pd}(\mathrm{dba})_{2} /\right.$ $\mathrm{dppf} / \mathrm{P}(\mathrm{OPh})_{3}$ 为催化剂, 甲苯为溶剂, $\left.55{ }^{\circ} \mathrm{C}\right]^{[8]}$ 应用于手 性烯丙型醇 1a $(99 \% e e)$ 与肼基甲酸酯 $\mathbf{2 a}$ 的烯丙型烷基 化反应，没有发现预期产物烯丙型肼 $\mathbf{3 a}$ 的生成. 于是, 我们改用 $2 \mathrm{~mol} \%[\mathrm{Pd}(\mathrm{allyl}) \mathrm{Cl}]_{2}$ 作为催化剂, 室温下在二 氧六环中笁选配体. 加入氧、氮配体的反应几乎不能进 行，而膦配体的结构显著影响反应效果(表 1, Entries 1 5 ). 用外消旋 BINAP 作为配体时, 反应 $3 \mathrm{~h}$ 后得到烯丙 型肼 3a 的产率仅为 $19 \%$, 双键的 $E$-构型得到保留，而对 映纯度略有降低(表 1, Entry 5). 我们更换了一些钯源, 但产率没有提高(表 1, Entries 6 9). 借鉴以前的研究工 作 ${ }^{[3 \mathrm{~b}, 6]}$, 我们加入 1 equiv. 嗍酸来活化烯丙型醇 $1 \mathbf{a}$ 的羟 基, 发现产率升至 $80 \%$, 而且立体构型得到完全保留(表 1, Entry 10). 但加入三氯化铁、二氯化锌、苯甲酸或者 对甲苯磺酸后, 不发生反应或者产率很低(表 1, Entries 11 14). 对照实验表明, 硼酸本身不能催化反应, 而且 降低用量会导致产率和对映纯度的降低(表 1, Entries 15, 16). 我们也尝试了一些其它常见有机溶剂, 发现产率 明显下降, 甚至不发生反应(表 1, Entries 17 23). 将温 度升至 $40{ }^{\circ} \mathrm{C}$, 产率提高到 $94 \%$, 但对映纯度略有降低 (表 1, Entry 24). 将反应时间延长至 $6 \mathrm{~h}$, 产率提高到
$83 \%$ ，而且立体构型得到完全保留(表 1, Entry 25). 当用 光学活性的 $(R)$-BINAP 代替外消旋 BINAP 时, 产率降低 至 46\%, 立体构型得到保留, 但对映纯度降至 97\%(表 1, Entry 26). 另一方面, 用 (S)-BINAP 作为配体时, 产率为 $82 \%$ ，立体构型得到完全保留(表 1, Entry 27). 这些结果 表明, 反应的立体化学由底物决定, 但反应速率受到手 性配体立体构型的显著影响. 综合考虑反应效果和实用 性, 我们决定采用比较便宜的外消旋 BINAP 作为配体.

在 $2 \mathrm{~mol} \%[\mathrm{Pd}(\mathrm{allyl}) \mathrm{Cl}]_{2} 、 4 \mathrm{~mol} \%$ 外消旋 BINAP 和 1 equiv. 嗍酸的作用下, 一系列高度对映富集的非对称 烯丙型醇与肼基甲酸酯 $\mathbf{2 a}$ 进行对映专一性烯丙型烷基 化反应，生成高对映纯度的烯丙型肼，具有很高的区域 选择性, 双键的 $E$-构型得到完全保留, 立体构型的保留 率 $[e e(\mathbf{3}) / e e(1) \times 100 \%]$ 介于 $98 \sim 100 \%$ (表 2, Entries 1 12). 大多数反应给出良好的产率，但含 $\alpha$-烷基和 $\gamma$-烷基 的烯丙型醇 $1 \mathrm{~h}$ 以及含较大空间位阻的 $\gamma$-取代基的烯丙 型醇 $\mathbf{1 k}$ 参与的反应给出的产率比较低(表 2 , Entries 8 , 11 ). 含 $\beta$-取代基的烯丙型醇, 例如 $(R, E)$-3-甲基-4-苯基 -3-丁烯- 2-醇, 参与的反应则非常缓慢. 上述反应的 $\alpha / \gamma$ 选择性取决于烯丙型醇的 $\alpha$-取代基和 $\gamma$-取代基的类型, $\mathrm{C}-\mathrm{N}$ 键的生成优先发生在将导致双键共轭以及较小空 间位阻的烯丙位. 当 $\alpha$-取代基与 $\gamma$-取代基相同时，反应 给出外消旋产物(表 2, Entry 13). $N^{\prime}$-取代肼基甲酸酯的 空间位阻使得反应活性降低, 在较高温度下才能与烯丙 型醇反应, 给出中等产率, 具有高度的区域选择性, 但 对映纯度降低 2 4\%(表 2, Entries 14，15). 其它肼基甲 酸酯、酰肼、磷酰肼等单取代肼都可以与手性烯丙型醇 进行对映专一性烯丙型烷基化反应，具有高度的区域选 择性，生成含多种保护基的手性烯丙型肼(表 2, Entries 16 20).

在前述反应条件下, 我们还尝试了另外两种单取代 肼, 但经过后处理所得到的产物均不是烯丙型肼. 烯丙 型醇 1a 与苯肼 $(2 \mathrm{i})$ 的反应混合物暴露在空气中, 其烯丙 型烷基化产物 $3 \mathrm{t}$ 不稳定 ${ }^{[14]}$, 容易被氧化成腙 4 , 产率为 $92 \%$ (Scheme 2). 另外, 烯丙型醇 $\mathbf{1 a}$ 与磺酰肼 $\mathbf{2 j}$ 反应而

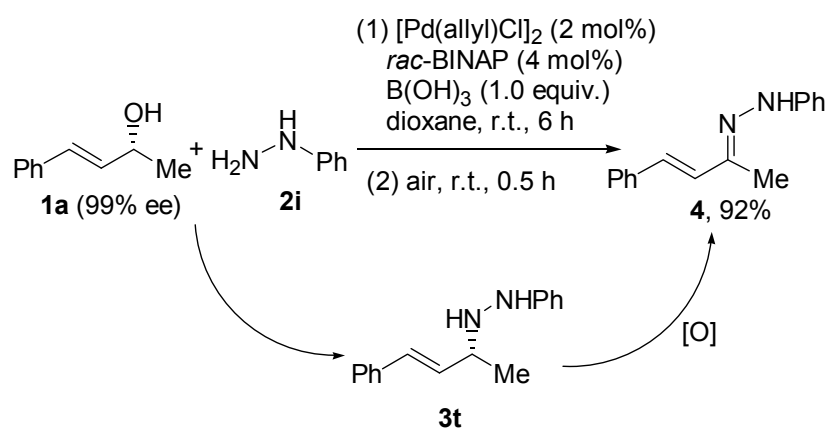

图式 2 与苯肼 $(2 \mathrm{i})$ 的反应

Scheme 2 Reaction with phenylhydrazine (2i) 
表 1 反应条件的优化 ${ }^{a}$

Table 1 Optimization of reaction conditions

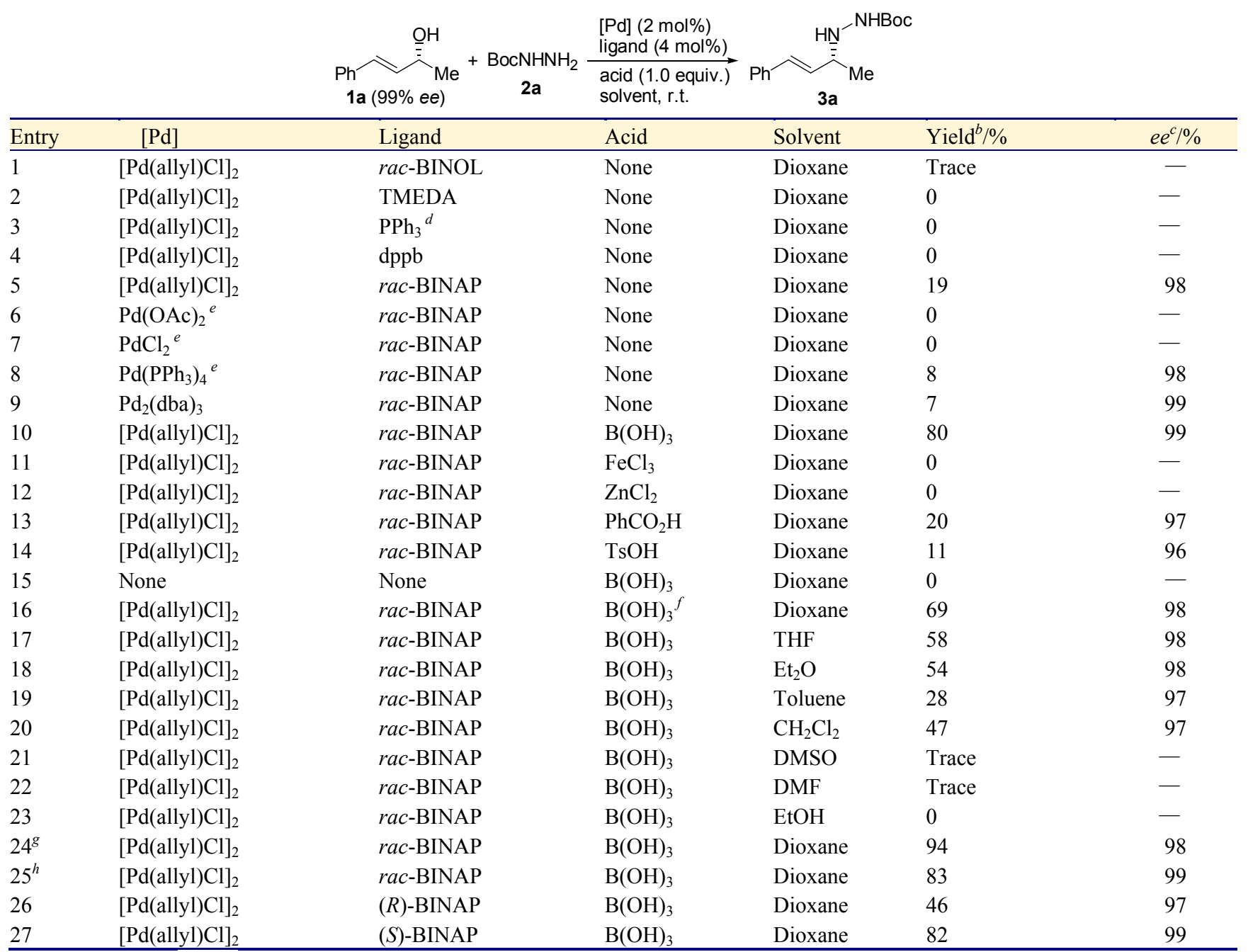

${ }^{a}$ Reaction conditions: Alcohol 1a $(0.20 \mathrm{mmol})$, carbazate $2 \mathrm{a}(0.24 \mathrm{mmol})$, [Pd] $(2 \mathrm{~mol} \%)$, ligand $(4 \mathrm{~mol} \%)$, acid $(0.20 \mathrm{mmol})$, solvent $(1.0 \mathrm{~mL})$, r.t., $3 \mathrm{~h} .{ }^{b}$ Isolated yield. ${ }^{c}$ Determined by HPLC analysis on a chiral stationary phase. ${ }^{d} 8 \mathrm{~mol} \% .{ }^{e} 4 \mathrm{~mol} \%{ }^{f} 0.10 \mathrm{mmol} .{ }^{g}$ The reaction was run at $40{ }^{\circ} \mathrm{C} .{ }^{h}$ The reaction was run for $6 \mathrm{~h}$. $\mathrm{BINAP}=2,2^{\prime}$-bis(diphenylphosphino)-1,1'-binaphthyl, BINOL $=1,1^{\prime}$-binaphthol, dba $=$ dibenzylideneacetone, dppb $=1,4$-bis(diphenylphosphino) butane, DMF $=$ $N, N$-dimethylformamide, $\mathrm{DMSO}=$ dimethyl sulfoxide, TMEDA $=N, N, N^{\prime}, N^{\prime}$-tetramethylethylenediamine, $\mathrm{THF}=$ tetrahydrofuran, $\mathrm{Ts}=p$-toluenesulfonyl.

生成的 $N^{\prime}$-烯丙基磺酰肼 $3 \mathbf{u}$ 也容易被空气氧化 ${ }^{[13 \mathrm{j}]}$, 经过 偶氮中间体 $6^{[17]}$, 分解成烯丙砜 5 , 产率仅为 $16 \%$ (Scheme 3). 改用 $10 \mathrm{~mol} \% \mathrm{Pd}(\mathrm{OAc})_{2}$ 和 $20 \mathrm{~mol} \%$ 外消旋 BINAP 来催化反应 ${ }^{[13 \mathrm{j}]}$, 同时不加入嗍酸, 产率提高到 $61 \%$, 但对映纯度降低 $9 \%$.

根据上述实验事实以及文献报道 ${ }^{[3 b, 6]}$, 我们提出如 下的反应机理来解释手性烯丙型醇与取代肼反应的立 体化学(图式 4). [Pd(allyl)Cl $]_{2}$ 被取代肼 2 和膦配体还原 成零价钯 $\left(\mathrm{PdL}_{n}\right)$. 烯丙型醇 $\mathbf{1}$ 的羟基由硼酸活化后, 受 到零价钯的背面进攻而脱掉羟基, 生成 $\pi$-烯丙基钯中间 体 8. 取代肼从背面进攻中间体 $\mathbf{8}$, 生成产物烯丙型肼 $\mathbf{3}$ 和副产物水, 同时释放零价钯和嘲酸, 进入下一个催化 循环圈. 在反应中烯丙型醇 1 的手性中心构型翻转了两 次, 因此最终得到构型保留的产物. 反应的区域选择性 取决于烯丙型醇 $\mathbf{1}$ 的 $\alpha$-取代基和 $\gamma$-取代基的电子和立体

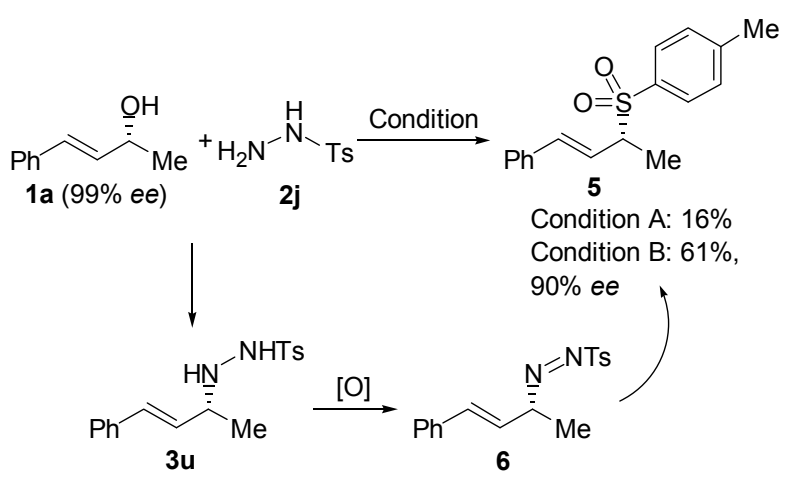

Condition A: $\left[\mathrm{Pd}(\text { allyl)Cl}]_{2}\right.$ (2 mol\%), rac-BINAP (4 mol\%), $\mathrm{B}(\mathrm{OH})_{3}$ (1 equiv.), dioxane, r.t., $6 \mathrm{~h}$. Condition B: Pd(OAc) $)_{2}(10 \mathrm{~mol} \%)$, rac-BINAP (20 mol\%), dioxane, air, r.t., $24 \mathrm{~h}$

图式 $\mathbf{3}$ 与磺酰肼 $\mathbf{2} \mathbf{j}$ 的反应

Scheme 3 Reaction with sulfonyl hydrazide $\mathbf{2 j}$ 
表 2 对映富集的烯丙型醇与取代肼的烯丙型烷基化反应 ${ }^{a}$

Table 2 Allylic alkylation of substituted hydrazines with enantioenriched allylic alcohols ${ }^{a}$

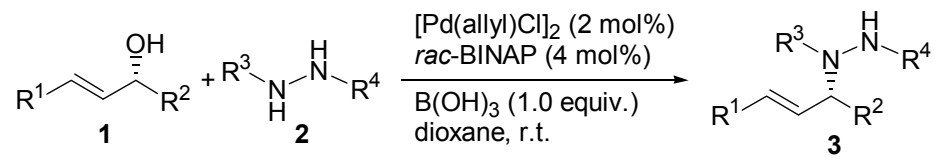

\begin{tabular}{|c|c|c|c|c|c|c|c|c|c|c|}
\hline \multirow{2}{*}{ Entry } & \multirow{2}{*}{1} & \multirow{2}{*}{$\mathrm{R}^{1}$} & \multirow{2}{*}{$\mathrm{R}^{2}$} & \multirow{2}{*}{2} & \multirow{2}{*}{$\mathrm{R}^{3}$} & \multirow{2}{*}{$\mathrm{R}^{4}$} & \multirow{2}{*}{3} & \multirow{2}{*}{ Yield $^{b} / \%$} & \multicolumn{2}{|c|}{$e e^{c} / \%$} \\
\hline & & & & & & & & & 1 & 3 \\
\hline 1 & $1 \mathrm{a}$ & $\mathrm{Ph}$ & $\mathrm{Me}$ & $2 a$ & $\mathrm{H}$ & Boc & $3 a$ & 83 & 99 & 99 \\
\hline 2 & $1 b$ & 4- $\mathrm{MeOC}_{6} \mathrm{H}_{4}$ & $\mathrm{Me}$ & $2 a$ & $\mathrm{H}$ & Boc & $3 b$ & 74 & 93 & 91 \\
\hline 3 & $1 \mathrm{c}$ & $4-\mathrm{ClC}_{6} \mathrm{H}_{4}$ & $\mathrm{Me}$ & $2 a$ & $\mathrm{H}$ & Boc & $3 c$ & 90 & 93 & 93 \\
\hline 4 & 1d & 2- $\mathrm{MeOC}_{6} \mathrm{H}_{4}$ & $\mathrm{Me}$ & $2 a$ & $\mathrm{H}$ & Boc & 3d & 65 & 94 & 92 \\
\hline 5 & $1 \mathrm{e}$ & 2-Naphthyl & $\mathrm{Me}$ & $2 a$ & $\mathrm{H}$ & Boc & $3 e$ & 75 & 92 & 92 \\
\hline 6 & 1f & 2-Furyl & $\mathrm{Me}$ & $2 a$ & $\mathrm{H}$ & Boc & $3 f$ & 84 & 95 & 95 \\
\hline 7 & $1 \mathrm{~g}$ & 2-Thienyl & $\mathrm{Me}$ & $2 a$ & $\mathrm{H}$ & Boc & $3 g$ & 75 & 90 & 89 \\
\hline 8 & $1 \mathrm{~h}$ & Cyclohexyl & $\mathrm{Me}$ & $2 a$ & $\mathrm{H}$ & Boc & $3 \mathrm{~h}$ & 25 & 96 & 96 \\
\hline 9 & $1 \mathrm{i}$ & $\mathrm{Ph}$ & Et & $2 a$ & $\mathrm{H}$ & Boc & $3 \mathbf{i}$ & 73 & 97 & 97 \\
\hline 10 & $1 \mathrm{j}$ & $\mathrm{Ph}$ & $\left(\mathrm{CH}_{2}\right)_{7} \mathrm{CH}_{3}$ & $2 a$ & $\mathrm{H}$ & Boc & $3 \mathbf{j}$ & 70 & 97 & 97 \\
\hline 11 & $1 \mathrm{k}$ & $\mathrm{Ph}$ & $\mathrm{CHMe}_{2}$ & $2 a$ & $\mathrm{H}$ & Boc & $3 \mathbf{k}$ & 31 & 95 & 95 \\
\hline 12 & 11 & $\mathrm{Me}$ & $\mathrm{Ph}$ & $2 a$ & $\mathrm{H}$ & Boc & ent $-\mathbf{3} \mathbf{a}^{d}$ & 80 & 95 & 95 \\
\hline 13 & $1 \mathrm{~m}$ & $\mathrm{Ph}$ & $\mathrm{Ph}$ & $2 a$ & $\mathrm{H}$ & Boc & 31 & 45 & 93 & 0 \\
\hline $14^{e}$ & $1 \mathrm{a}$ & $\mathrm{Ph}$ & $\mathrm{Me}$ & $2 b$ & Allyl & Boc & $3 m$ & 50 & 99 & 95 \\
\hline $15^{e}$ & $1 \mathrm{a}$ & $\mathrm{Ph}$ & $\mathrm{Me}$ & $2 c$ & $\mathrm{Bn}$ & Boc & $3 n$ & 67 & 99 & 97 \\
\hline 16 & $1 \mathrm{a}$ & $\mathrm{Ph}$ & $\mathrm{Me}$ & 2d & $\mathrm{H}$ & $\mathrm{CO}_{2} \mathrm{Et}$ & 30 & 93 & 99 & 99 \\
\hline 17 & $1 \mathrm{a}$ & $\mathrm{Ph}$ & $\mathrm{Me}$ & $2 e$ & $\mathrm{H}$ & $\mathrm{CO}_{2} \mathrm{Bn}$ & $3 p$ & 82 & 99 & 99 \\
\hline 18 & $1 \mathrm{a}$ & $\mathrm{Ph}$ & $\mathrm{Me}$ & $2 f$ & $\mathrm{H}$ & $\mathrm{COMe}$ & $3 q$ & 62 & 99 & 99 \\
\hline 19 & $1 \mathrm{a}$ & $\mathrm{Ph}$ & $\mathrm{Me}$ & $2 \mathrm{~g}$ & $\mathrm{H}$ & $\mathrm{COPh}$ & $3 \mathbf{r}$ & 76 & 99 & 99 \\
\hline 20 & $1 \mathrm{a}$ & $\mathrm{Ph}$ & $\mathrm{Me}$ & $2 \mathrm{~h}$ & $\mathrm{H}$ & $\mathrm{PO}(\mathrm{OEt})_{2}$ & $3 s$ & 50 & 99 & 97 \\
\hline
\end{tabular}

${ }^{a}$ Reaction conditions: Alcohol $1(0.20 \mathrm{mmol})$, substituted hydrazine $2(0.24 \mathrm{mmol})$, [Pd(allyl)Cl $]_{2}(2 \mathrm{~mol} \%)$, rac-BINAP $(4 \mathrm{~mol} \%), \mathrm{B}(\mathrm{OH})_{3}(0.20 \mathrm{mmol})$, dioxane $(1.0 \mathrm{~mL})$, r.t., 6 h. ${ }^{b}$ Isolated yield. ${ }^{c}$ Determined by HPLC analysis on a chiral stationary phase. ${ }^{d}$ Enantiomer of $3 \mathrm{3a} .{ }^{e}$ The reaction was run at $80{ }^{\circ} \mathrm{C}$ for $3 \mathrm{~h}$.

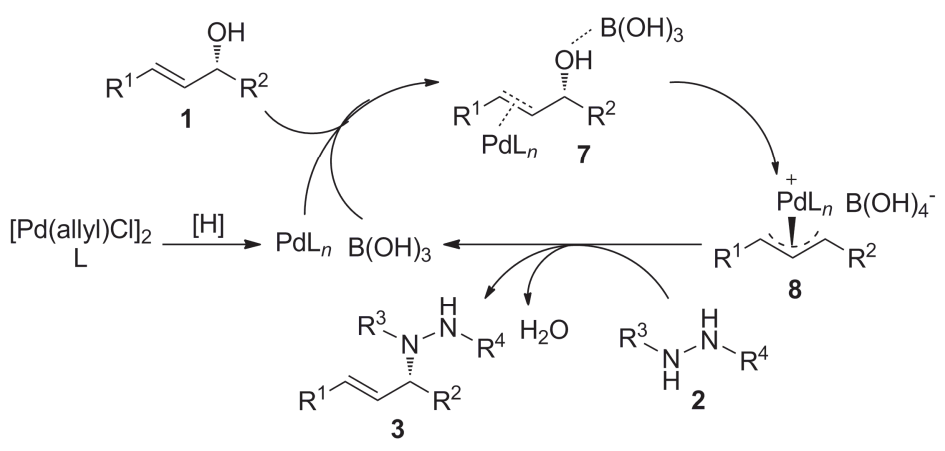

图式 4 建议的反应机理

Scheme 4 Proposed reaction mechanism

性质. $\pi$-烯丙基钯中间体 $\mathbf{8}$ 与取代肼 $\mathbf{2}$ 的 $\mathrm{C}-\mathrm{N}$ 键生成优 先发生在将导致双键共轭以及较小空间位阻的烯丙位. 当烯丙型醇 1 的 $\alpha$-取代基与 $\gamma$-取代基相同时, 取代肼 $\mathbf{2}$ 进攻中间体 8 的 $\alpha$-位和 $\gamma$-位的机会是均等的, 将给出等 量的对映体, 导致手性的完全丢失.

\section{2 结论}

我们发展了首例烯丙型醇与取代肼的对映专一性 烯丙型烷基化反应. 在 $2 \mathrm{~mol} \%\left[\mathrm{Pd}(\text { allyl)Cl}]_{2} 、 4 \mathrm{~mol} \%\right.$ 外
消旋 BINAP 和 1 equiv. 嗍酸的作用下，一系列高度对映 富集的烯丙型醇与取代肼在室温进行烯丙型烷基化反 应, 具有高度的区域选择性, 立体构型的保留率大于 $95 \%$, 是合成高对映纯度的烯丙型肼的有效方法. 另外, 该反应的副产物是水, 对环境友好.

\section{3 实验部分}

\section{1 仪器与试剂}

核磁共振氢谱和碳谱 $\left({ }^{1} \mathrm{H}\right.$ NMR 和 $\left.{ }^{13} \mathrm{C} \mathrm{NMR}\right)$ 由 
Bruker AC-400 FT 型核磁共振仪(分别为 $400 \mathrm{MHz}$ 和 100 $\mathrm{MHz}$ )测定, 以四甲基硅烷为内标. 高分辨质谱(HRMS) 由配备电喷雾电离(ESI)源的 Thermo Orbitrap XL 型质谱 仪测定. 对映体过量(ee)值由高压液相色谱(HPLC)测定: 惠普 1200 系列高压液相色谱仪, 配备大赛璐手性柱(IC、 $\mathrm{AS}$ 或者 $\mathrm{AD}, 250 \times 4.6 \mathrm{~mm})$, 用正己烷/异丙醇作为流动 相, 紫外光谱 $(U V)$ 检测波长 $(\lambda)$ 为 $254 \mathrm{~nm}$ 或 $220 \mathrm{~nm}$. 旋 光度由 Perkin-Elmer 343 型偏光计在钠灯 $589 \mathrm{~nm}$ 下测 定. 熔点由显微熔点仪测定，未经校正.

手性烯丙型醇 $\mathbf{1 a} \sim \mathbf{1 m}$ 都是已知物, 由 Sharpless 不 对称环氧化反应动力学拆分其外消旋体而制得 ${ }^{[18]}$. 取代 肼 2b 和 2c 可按照文献[19]方法而制得. 其它药品在购 买后直接使用.

\section{2 实验方法}

手性烯丙型醇 $1(0.20 \mathrm{mmol})$ 、取代肼 $2(0.24 \mathrm{mmol})$ 、 $[\mathrm{Pd}(\text { allyl }) \mathrm{Cl}]_{2}(1.5 \mathrm{mg}, 2 \mathrm{~mol} \%)$ 、外消旋 BINAP (5.0 mg, 4 $\mathrm{mol} \%$ )、嗍酸 $(12.4 \mathrm{mg}, 0.20 \mathrm{mmol})$ 和二氧六环 $(1.0 \mathrm{~mL})$ 的混合物在氮气氛围下于室温搅拌 $6 \mathrm{~h}$. 反应混合物直 接经硅胶层析纯化 $[V$ (二氯甲烷 $): V($ 石油醚 $)=1 ： 0 \sim$ 1:1], 得到手性烯丙型肼 3 .

与文献记载的比旋光度相比较, 确定了产物 $3 \mathbf{a}$ 、 ent $-3 \mathrm{a} 、 3 \mathrm{e} 、 3 \mathrm{~h} 、 3 \mathrm{i} 、 3 \mathrm{~m} \sim 3 \mathrm{~s}$ 和 5 的绝对立体构型. 类 推而得其它产物的绝对立体构型.

$(R, E)-2$-(4-甲氧基苯基-3-丁烯-2-基)肼基甲酸叔丁 酯(3b): 得 $43.3 \mathrm{mg}$ 白色粉末, 产率 $74 \%, 91 \%$ ee. m.p. $82 \sim 84{ }^{\circ} \mathrm{C} ;[\alpha]_{\mathrm{D}}^{20}+112\left(c \quad 1.0, \mathrm{CHCl}_{3}\right) ;{ }^{1} \mathrm{H}$ NMR $(400$ $\left.\mathrm{MHz}, \mathrm{CDCl}_{3}\right) \delta: 7.30(\mathrm{~d}, J=8.7 \mathrm{~Hz}, 2 \mathrm{H}), 6.85(\mathrm{~d}, J=8.7$ $\mathrm{Hz}, 2 \mathrm{H}), 6.45$ (d, $J=15.8 \mathrm{~Hz}, 1 \mathrm{H}), 6.09$ (br s, 1H), 5.91 $(\mathrm{dd}, J=15.8,8.1 \mathrm{~Hz}, 1 \mathrm{H}), 3.80(\mathrm{~s}, 3 \mathrm{H}), 3.75 \sim 3.65(\mathrm{~m}$, 1H), 1.44 (s, 9H), 1.20 (d, $J=6.4 \mathrm{~Hz}, 3 \mathrm{H}) ;{ }^{13} \mathrm{C}$ NMR $(100$ $\left.\mathrm{MHz}, \mathrm{CDCl}_{3}\right) \delta: 159.3,156.9,131.5,129.8,129.4,127.7$, 114.1, 80.6, 58.3, 55.4, 28.5, 19.4; HRMS (ESI) calcd for $\mathrm{C}_{16} \mathrm{H}_{25} \mathrm{O}_{3} \mathrm{~N}_{2}(\mathrm{M}+\mathrm{H})^{+}$293.1860, found 293.1859; ee 值测 定条件: Chiralpak AD 柱, $V$ (正己烷) : $V$ (异丙醇 $)=90$ : 10 , 流速 $=1.0 \mathrm{~mL} / \mathrm{min}, \mathrm{UV}$ 检测 $\lambda=254 \mathrm{~nm}, t_{\mathrm{R}}=14.4$ $\min$ (次要), $17.2 \min$ (主要).

$(R, E)$-2-(4-氯苯基-3-丁烯-2-基)肼基甲酸叔丁酯 (3c): 得 $53.4 \mathrm{mg}$ 白色粉末, 产率 $90 \%, 93 \%$ ee. m.p. 91 $93{ }^{\circ} \mathrm{C} ;[\alpha]_{\mathrm{D}}^{20}+142\left(c 1.0, \mathrm{CHCl}_{3}\right) ;{ }^{1} \mathrm{H}$ NMR $(400 \mathrm{MHz}$, $\left.\mathrm{CDCl}_{3}\right) \delta: 7.31 \sim 7.23(\mathrm{~m}, 4 \mathrm{H}), 6.45(\mathrm{~d}, J=15.9 \mathrm{~Hz}, 1 \mathrm{H})$, 6.15 (s, br, 1H), 6.04 (dd, $J=15.9,8.0 \mathrm{~Hz}, 1 \mathrm{H}), 3.77 \sim 3.65$ $(\mathrm{m}, 1 \mathrm{H}), 1.44(\mathrm{~s}, 9 \mathrm{H}), 1.20(\mathrm{~d}, J=6.5 \mathrm{~Hz}, 3 \mathrm{H}) ;{ }^{13} \mathrm{C} \mathrm{NMR}$ $\left(100 \mathrm{MHz}, \mathrm{CDCl}_{3}\right) \delta: 156.9,135.6,133.2,132.5,130.5$, 128.8, 127.6, 80.7, 58.2, 28.5, 19.2; HRMS (ESI) calcd for $\mathrm{C}_{15} \mathrm{H}_{22} \mathrm{O}_{2} \mathrm{~N}_{2} \mathrm{Cl}(\mathrm{M}+\mathrm{H})^{+}$297.1364, found 297.1362; ee 值
测定条件: Chiralpak $\mathrm{AD}$ 柱, $V$ (正己烷) $: V$ (异丙醇) $=$ $95: 5$, 流速 $=1.0 \mathrm{~mL} / \mathrm{min}, \mathrm{UV}$ 检测 $\lambda=254 \mathrm{~nm}, t_{\mathrm{R}}=11.7$ $\min$ (次要), $13.5 \min$ (主要).

$(R, E)$-2-(2-甲氧基苯基-3-丁烯-2-基)肼基甲酸叔丁 酯(3d): 得 $38.0 \mathrm{mg}$ 无色油状物, 产率 $65 \%, 92 \%$ ee. $[\alpha]_{\mathrm{D}}^{20}+96\left(c\right.$ 1.0, $\left.\mathrm{CHCl}_{3}\right) ;{ }^{1} \mathrm{H}$ NMR $\left(400 \mathrm{MHz}, \mathrm{CDCl}_{3}\right) \delta$ : $7.44(\mathrm{dd}, J=7.6,1.4 \mathrm{~Hz}, 1 \mathrm{H}), 7.24 \sim 7.18(\mathrm{~m}, 1 \mathrm{H}), 6.93 \sim$ $6.81(\mathrm{~m}, 3 \mathrm{H}), 6.11$ (br s, $1 \mathrm{H}), 6.06(\mathrm{dd}, J=16.0,8.1 \mathrm{~Hz}$, $1 \mathrm{H}), 3.84(\mathrm{~s}, 3 \mathrm{H}), 3.79 \sim 3.69(\mathrm{~m}, 1 \mathrm{H}), 1.45(\mathrm{~s}, 9 \mathrm{H}), 1.21$ $(\mathrm{d}, J=6.4 \mathrm{~Hz}, 3 \mathrm{H}) ;{ }^{13} \mathrm{C} \mathrm{NMR}\left(100 \mathrm{MHz}, \mathrm{CDCl}_{3}\right) \delta: 156.8$, 132.1, 128.7, 126.9, 126.6, 126.0, 120.7, 111.0, 80.5, 58.5, 55.5, 28.3, 19.4; HRMS (ESI) calcd for $\mathrm{C}_{16} \mathrm{H}_{25} \mathrm{O}_{3} \mathrm{~N}_{2}(\mathrm{M}+$ $\mathrm{H})^{+}$293.1860, found 293.1856; ee 值测定条件: Chiralpak $\mathrm{AD}$ 柱, $V$ (正己烷 $): V$ (异丙醇 $)=90 ： 10$, 流速 $=1.0$ $\mathrm{mL} / \mathrm{min}, \mathrm{UV}$ 检测 $\lambda=254 \mathrm{~nm}, t_{\mathrm{R}}=11.2 \min$ (次要), 14.2 $\min$ (主要).

$(R, E)$-2-[4-(2-呋喃基)-3-丁烯-2-基]肼基甲酸叔丁酯 (3f): 得 $42.4 \mathrm{mg}$ 白色粉末, 产率 84\%, 95\% ee. m.p. 80 $82{ }^{\circ} \mathrm{C} ;[\alpha]_{\mathrm{D}}^{20}+145\left(\right.$ c $\left.1.0, \mathrm{CHCl}_{3}\right) ;{ }^{1} \mathrm{H}$ NMR (400 MHz, $\left.\mathrm{CDCl}_{3}\right) \delta: 7.34 \sim 7.32(\mathrm{~m}, 1 \mathrm{H}), 6.37 \sim 6.31(\mathrm{~m}, 2 \mathrm{H}), 6.23 \sim$ $6.20(\mathrm{~m}, 2 \mathrm{H}), 6.00(\mathrm{dd}, J=15.9,8.1 \mathrm{~Hz}, 1 \mathrm{H}), 3.74 \sim 3.65$ (m, 1H), 3.43 (s, br, 1H), 1.44 (s, 9H), 1.19 (d, $J=6.5 \mathrm{~Hz}$, $3 \mathrm{H}) ;{ }^{13} \mathrm{C} \mathrm{NMR}\left(100 \mathrm{MHz}, \mathrm{CDCl}_{3}\right) \delta: 156.9,152.6,142.0$, 130.4, 120.2, 111.2, 107.8, 80.6, 57.9, 28.4, 19.3; HRMS (ESI) calcd for $\mathrm{C}_{13} \mathrm{H}_{21} \mathrm{O}_{3} \mathrm{~N}_{2}(\mathrm{M}+\mathrm{H})^{+}$253.1547, found 253.1544; $e e$ 值测定条件: Chiralpak AD 柱, $V$ (正己烷) : $V($ 异丙醇 $)=90: 10$, 流速 $=1.0 \mathrm{~mL} / \mathrm{min}, \mathrm{UV}$ 检测 $\lambda=254$ $\mathrm{nm}, t_{\mathrm{R}}=6.7 \mathrm{~min}$ (次要), $7.7 \mathrm{~min}$ (主要).

$(R, E)$-2-[4-(2-噻吩基)-3-丁烯-2-基]肼基甲酸叔丁酯 $(3 \mathrm{~g})$ : 得 $40.3 \mathrm{mg}$ 淡黄色油状物, 产率 $75 \%, 89 \%$ ee. m.p. 97 $98{ }^{\circ} \mathrm{C} ;[\alpha]_{\mathrm{D}}^{20}+121\left(c 1.0, \mathrm{CHCl}_{3}\right) ;{ }^{1} \mathrm{H}$ NMR $(400$ $\left.\mathrm{MHz}, \mathrm{CDCl}_{3}\right) \delta: 7.14(\mathrm{dd}, J=4.7,0.8 \mathrm{~Hz}, 1 \mathrm{H}), 6.97 \sim 6.91$ $(\mathrm{m}, 2 \mathrm{H}), 6.64(\mathrm{~d}, J=15.7 \mathrm{~Hz}, 1 \mathrm{H}), 6.10$ (br s, 1H), 5.89 $(\mathrm{dd}, J=15.7,8.0 \mathrm{~Hz}, 1 \mathrm{H}), 3.74 \sim 3.63(\mathrm{~m}, 1 \mathrm{H}), 1.45(\mathrm{~s}$, 9H), $1.20(\mathrm{~d}, J=6.5 \mathrm{~Hz}, 3 \mathrm{H}) ;{ }^{13} \mathrm{C} \mathrm{NMR}\left(100 \mathrm{MHz}, \mathrm{CDCl}_{3}\right)$ $\delta: 156.9,142.2,131.3,127.4,125.7,125.0,124.3,80.7$, 58.1, 28.5, 19.4; HRMS (ESI) calcd for $\mathrm{C}_{13} \mathrm{H}_{21} \mathrm{O}_{2} \mathrm{~N}_{2} \mathrm{~S}(\mathrm{M}+$ $\mathrm{H})^{+}$269.1318, found 269.1318; ee 值测定条件: Chiralpak $\mathrm{AD}$ 柱, $V$ (正己烷 $): V$ (异丙醇 $)=90 ： 10$, 流速 $=1.0$ $\mathrm{mL} / \mathrm{min}, \mathrm{UV}$ 检测 $\lambda=254 \mathrm{~nm}, t_{\mathrm{R}}=8.7 \mathrm{~min}$ (次要), 10.4 $\min$ (主要).

$(R, E)$-2-(1-苯基-1-十一碳烯-3-基)肼基甲酸叔丁酯 (3j): 得 $50.5 \mathrm{mg}$ 白色固体，产率 70\%, 97\% ee. m.p. 59 $60{ }^{\circ} \mathrm{C} ;[\alpha]_{\mathrm{D}}^{20}+178\left(\right.$ c $\left.1.0, \mathrm{CHCl}_{3}\right) ;{ }^{1} \mathrm{H}$ NMR $(400 \mathrm{MHz}$, $\left.\mathrm{CDCl}_{3}\right) \delta: 7.40 \sim 7.35(\mathrm{~m}, 2 \mathrm{H}), 7.30(\mathrm{t}, J=7.5 \mathrm{~Hz}, 2 \mathrm{H})$, 
$7.25 \sim 7.20(\mathrm{~m}, 1 \mathrm{H}), 6.49(\mathrm{~d}, J=15.9 \mathrm{~Hz}, 1 \mathrm{H}), 6.15$ (br s, $1 \mathrm{H}), 5.99$ (dd, $J=15.9,8.6 \mathrm{~Hz}, 1 \mathrm{H}), 3.60 \sim 3.49(\mathrm{~m}, 1 \mathrm{H})$, 3.41 (br s, $1 \mathrm{H}), 1.64 \sim 1.52(\mathrm{~m}, 1 \mathrm{H}), 1.43(\mathrm{~s}, 9 \mathrm{H}), 1.37 \sim$ $1.17(\mathrm{~m}, 13 \mathrm{H}), 0.87(\mathrm{t}, J=6.8 \mathrm{~Hz}, 3 \mathrm{H}) ;{ }^{13} \mathrm{C} \mathrm{NMR}(100$ $\left.\mathrm{MHz}, \mathrm{CDCl}_{3}\right) \delta: 156.8,137.1,133.0,130.8,128.7,127.6$, 126.5, 80.5, 63.6, 33.45, 32.1, 29.9, 29.6, 29.4, 28.5, 26.0, 22.8, 14.2; HRMS (ESI) calcd for $\mathrm{C}_{22} \mathrm{H}_{37} \mathrm{O}_{2} \mathrm{~N}_{2}(\mathrm{M}+\mathrm{H})^{+}$ 361.2850, found 361.2847; ee 值测定条件: Chiralpak AD 柱, $V$ (正己烷) $: V$ (异丙醇 $)=95: 5$, 流速 $=1.0 \mathrm{~mL} / \mathrm{min}$, $\mathrm{UV}$ 检测 $\lambda=254 \mathrm{~nm}, t_{\mathrm{R}}=7.5 \mathrm{~min}$ (次要), $6.8 \mathrm{~min}$ (主要).

$(R, E)$-2-(1-苯基-4-甲基-1-戊烯-3-基)肼基甲酸叔丁 酯(3k): 得 $18.0 \mathrm{mg}$ 淡黄色油状物, 产率 $31 \%, 95 \%$ ee. $[\alpha]_{\mathrm{D}}^{20}+94\left(c 1.0, \mathrm{CHCl}_{3}\right) ;{ }^{1} \mathrm{H}$ NMR $\left(400 \mathrm{MHz}, \mathrm{CDCl}_{3}\right) \delta$ : 7.39 (d, $J=7.3 \mathrm{~Hz}, 2 \mathrm{H}), 7.34 \sim 7.29(\mathrm{~m}, 2 \mathrm{H}), 7.26 \sim 7.20$ (m, 1H), 6.49 (d, $J=15.9 \mathrm{~Hz}, 1 \mathrm{H}), 6.03$ (dd, $J=15.9,8.8$ $\mathrm{Hz}, 2 \mathrm{H}), 3.44 \sim 3.32(\mathrm{~m}, 1 \mathrm{H}), 1.90 \sim 1.76(\mathrm{~m}, 1 \mathrm{H}), 1.43(\mathrm{~s}$, 9H), $1.00(\mathrm{~d}, J=6.8 \mathrm{~Hz}, 3 \mathrm{H}), 0.94(\mathrm{~d}, J=6.8 \mathrm{~Hz}, 3 \mathrm{H}) ;{ }^{13} \mathrm{C}$ NMR $\left(100 \mathrm{MHz}, \mathrm{CDCl}_{3}\right) \delta: 156.8,137.1,133.9,128.7$, 128.4, 127.6, 126.5, 80.5, 69.1, 30.8, 28.5, 19.7, 18.2; HRMS (ESI) calcd for $\mathrm{C}_{17} \mathrm{H}_{27} \mathrm{O}_{2} \mathrm{~N}_{2}(\mathrm{M}+\mathrm{H})^{+}$291.2067, found 291.2065; $e e$ 值测定条件: Chiralpak AD 柱, $V$ (正己 烷 $): V$ (异丙醇 $)=90: 10$, 流速 $=1.0 \mathrm{~mL} / \mathrm{min}, \mathrm{UV}$ 检测 $\lambda=254 \mathrm{~nm}, t_{\mathrm{R}}=5.4 \mathrm{~min}$ (次要), $5.8 \mathrm{~min}$ (主要).

辅助材料(Supporting Information) 核磁共振图谱以 及高压液相色谱图. 这些材料可以免费从本刊网站 (http://sioc-journal.cn/)上下载.

\section{References and note}

[1] For reviews, see:

(a) Tsuji, J. Acc. Chem. Res. 1969, 2, 144.

(b) Trost, B. M. Tetrahedron 1977, 33, 2615.

(c) Trost, B. M.; Van Vranken, D. L. Chem. Rev. 1996, 96, 395.

(d) Trost, B. M.; Crawley, M. L. Chem. Rev. 2003, 103, 2921.

(e) Kazmaier, U.; Pohlman, M. In Metal-Catalyzed Cross-Coupling Reactions, 2nd ed., Eds.: de Meijere, A.; Diederich, F., Wiley-VCH, Weinheim, 2004, p. 531.

[2] For reviews, see:

(a) Muzart, J. Tetrahedron 2005, 61, 4179.

(b) Bandini, M. Angew. Chem., Int. Ed. 2011, 50, 994.

(c) Sundararaju, B.; Achard, M.; Bruneau, C. Chem. Soc. Rev. 2012, $41,4467$.

(d) Bandini, M.; Cera, G.; Chiarucci, M. Synthesis 2012, 44, 504.

[3] (a) Ye, J.; Zhao, J.; Xu, J.; Mao, Y.; Zhang, Y. J. Chem. Commun. 2013, 49, 9761.

(b) Wu, H.-B.; Ma, X.-T.; Tian, S.-K. Chem. Commun. 2014, 50, 219.

[4] (a) Ozawa, F.; Okamoto, H.; Kawagishi, S.; Yamamoto, S.; Minami, T.; Yoshifuji, M. J. Am. Chem. Soc. 2002, 124, 10968.

(b) Roggen, M.; Carreira, E. M. J. Am. Chem. Soc. 2010, 132, 11917. (c) Mukherjee, P.; Widenhoefer, R. A. Org. Lett. 2010, 12, 1184. For intramolecular substitution, see:

(d) Kawai, N.; Abe, R.; Uenishi, J. Tetrahedron Lett. 2009, 50, 6580.

(e) Hande, S. M.; Kawai, N.; Uenishi, J. J. Org. Chem. 2009, 74, 244.

(f) Kawai, N.; Abe, R.; Matsuda, M.; Uenishi, J. J. Org. Chem. 2011, 76, 2102.

(g) Mukherjee, P.; Widenhoefer, R. A. Org. Lett. 2011, 13, 1334.

(h) Mukherjee, P.; Widenhoefer, R. A. Angew. Chem., Int. Ed. 2012, 51, 1405.

[5] (a) Vikhe, Y. S.; Hande, S. M.; Kawai, N.; Uenishi, J. J. Org. Chem. 2009, 74, 5174.

(b) Mukherjee, P.; Widenhoefer, R. A. Chem.-Eur. J. 2013, 19, 3437.

For intramolecular substitution, see:

(c) Uenishi, J.; Ohmi, M.; Ueda, A. Tetrahedronn: Asymmetry 2005, 16, 1299.

(d) Kawai, N.; Lagrange, J.-M.; Ohmi, M.; Uenishi, J. J. Org. Chem. 2006, 71, 4530.

(e) Kawai, N.; Lagrange, J.-M.; Uenishi, J. Eur. J. Org. Chem. 2007, 2808.

(f) Uenishi, J.; Vikhe, Y. S.; Kawai, N. Chem. Asian J. 2008, 3, 473.

(g) Guérinot, A.; Serra-Muns, A.; Bensoussan, C.; Reymond, S.; Cossy, J. Tetrahedron 2011, 67, 5024.

(h) Unsworth, W. P.; Stevens, K.; Lamont, S. G.; Robertson, J. Chem. Commun. 2011, 47, 7659.

(i) Ghebreghiorgis, T.; Biannic, B.; Kirk, B. H.; Ess, D. H.; Aponick, A. J. Am. Chem. Soc. 2012, 134, 16307.

(j) Ghebreghiorgis, T.; Kirk, B. H.; Aponick, A.; Ess, D. H. J. Org. Chem. 2013, 78, 7664.

[6] Ma, X.-T.; Dai, R.-H.; Zhang, J.; Gu, Y.; Tian, S.-K. Adv. Synth. Catal. 2014, 356, 2984.

[7] Lumbroso, A.; Cooke, M. L.; Breit, B. Angew. Chem., Int. Ed. 2013, 52, 1890.

[8] Tšupova, S.; Mäeorg, U. Org. Lett. 2013, 15, 3381.

[9] (a) Zhang, R.; Durkin, J. P.; Windsor, W. T. Bioorg. Med. Chem. Lett. 2002, 12, 1005.

(b) Raja, A.; Lebbos, J.; Kirkpatrick, P. Nat. Rev. Drug Discovery 2003, 1,857 .

[10] For reviews, see:

(a) Ragnarsson, U. Chem. Soc. Rev. 2001, 30, 205.

(b) Friestad, G. K. Eur. J. Org. Chem. 2005, 3157.

[11] (a) Strick, B. F.; Mundal, D. A.; Thomson, R. J. J. Am. Chem. Soc. 2011, 133, 14252.

(b) Lutz, K. E.; Thomson, R. J. Angew. Chem., Int. Ed. 2011, 50, 4437.

(c) Gutierrez, O.; Strick, B. F.; Thomson, R. J.; Tantillo, D. J. Chem. Sci. 2013, 4, 3997.

(d) Reddel, J. C. T.; Lutz, K. E.; Diagne, A. B.; Thomson, R. J. Angew. Chem., Int. Ed. 2014, 53, 1395.

[12] (a) Yamagishi, T.; Ohnuki, M.; Kiyooka, T.; Masui, D.; Sato, K.; Yamaguchi, M. Tetrahedron: Asymmetry 2003, 14, 3275.

(b) Kloetzing, R. J.; Knochel, P. Tetrahedron: Asymmetry 2006, 17, 116.

(c) Popa, D.; Marcos, R.; Sayalero, S.; Vidal-Ferran, A.; Pericàs, M. A. Adv. Synth. Catal. 2009, 351, 1539.

(d) Yuan, H.; Zhou, Z.; Xiao, J.; Liang, L.; Dai, L. Tetrahedron: Asymmetry 2010, 21, 1874.

[13] (a) Li, H.-H.; Dong, D.-J.; Tian, S.-K. Eur. J. Org. Chem. 2008, 3623. 
(b) Li, H.-H.; Jin, Y.-H.; Wang, J.-Q.; Tian, S.-K. Org. Biomol. Chem. 2009, 7, 3219.

(c) Yang, C.-F.; Shen, C.; Li, H.-H.; Tian, S.-K. Chin. Sci. Bull. 2012, 57, 2377.

(d) Tian, Y.; Sui, Y.; Gu, Y.; Tian, S.-K. Adv. Synth. Catal. 2012, 354, 3475.

(e) Li, M.-B.; Wang, Y.; Tian, S.-K. Angew. Chem., Int. Ed. 2012, 51, 2968.

(f) Wu, X.-S.; Chen, Y.; Li, M.-B.; Zhou, M.-G.; Tian, S.-K. J. Am. Chem. Soc. 2012, 134, 14694.

(g) Li, M.-B.; Li, H.; Wang, J.; Liu, C.-R.; Tian, S.-K. Chem. Commun. 2013, 49, 8190.

(h) Ma, X.-T.; Wang, Y.; Dai, R.-H.; Liu, C.-R.; Tian, S.-K. J. Org. Chem. 2013, 78, 11071.

(i) Wu, X.-S.; Zhou, M.-G.; Chen, Y.; Tian, S.-K. Asian J. Org. Chem. 2014, 3, 711 .

(j) Wang, T.-T.; Wang, F.-X.; Yang, F.-L.; Tian, S.-K. Chem. Commun. 2014, 50, 3802.

(k) Zhou, M.-G.; Zhang, W.-Z.; Tian, S.-K. Chem. Commun. 2014, $50,14531$.

(1) Wang, Y.; Li, M.; Ma, X.; Liu, C.; Gu, Y.; Tian, S.-K. Chin. J. Chem. 2014, 32, 741.

[14] Wang, Y.; Xu, J.-K.; Gu, Y.; Tian, S.-K. Org. Chem. Front. 2014,
1,812 .

[15] (a) Bertelsen, S.; Marigo, M.; Brandes, S.; Dinér, P.; Jørgensen, K. A. J. Am. Chem. Soc. 2006, 128, 12973.

(b) Wang, J.; Chen, J.; Kee, C. W.; Tan, C.-H. Angew. Chem., Int. Ed. 2012, 51, 2382.

[16] (a) Yang, F.-L.; Ma, X.-T.; Tian, S.-K. Chem.-Eur. J. 2012, 18, 1582.

(b) Yang, F.-L.; Tian, S.-K. Angew. Chem., Int. Ed. 2013, 52, 4929.

(c) Su, Y.-H.; Wu, Z.; Tian, S.-K. Chem. Commun. 2013, 49, 6528.

(d) Zhang, Y.-G.; Liu, X.-L.; He, Z.-Y.; Li, X.-M.; Kang, H.-J.; Tian, S.-K. Chem.-Eur. J. 2014, 20, 2765.

(e) Yang, F.-L.; Wang, F.-X.; Wang, T.-T.; Wang, Y.-J.; Tian, S.-K. Chem. Commun. 2014, 50, 2111.

[17] Electrospray ionization (ESI) mass spectrometric analysis of the reaction mixture permitted us to assign intermediate $\mathbf{6}$ according to the high resolution mass data. HRMS (ESI) calcd for $\mathrm{C}_{17} \mathrm{H}_{18} \mathrm{~N}_{2} \mathrm{O}_{2} \mathrm{SNa}(\mathrm{M}+\mathrm{Na})^{+}$337.0981, found 337.0987.

[18] Martin, V. S.; Woodard, S. S.; Katsuki, T.; Yamada. Y.; Ikeda, M.; Sharpless, K. B. J. Am. Chem. Soc. 1981, 103, 6237.

[19] (a) Mäeorg, U.; Bredihhin, A. Org. Lett. 2007, 9, 4975.

(b) Zhang, Z.; Wang, M.; Yao, X.; Li, Y.; Tan, J.; Wang, L.; Qiao, W.; Geng, Y.; Liu, Y.; Wang, Q. Eur. J. Med. Chem. 2012, 54, 33.

(Cheng, F.) 